Document downloaded from:

http://hdl.handle.net/10251/57016

This paper must be cited as:

Gómez Lozano, V.; Rubio Michavila, C.; Candelas Valiente, P.; Belmar Ibáñez, F.; Uris Martínez, A. (2015). Geometrical isotropy in perforated plates with subwavelength holes decorated with Archimedean patterns. EPL. 111(3):34002p1-34002p5. doi:10.1209/0295$5075 / 111 / 34002$.

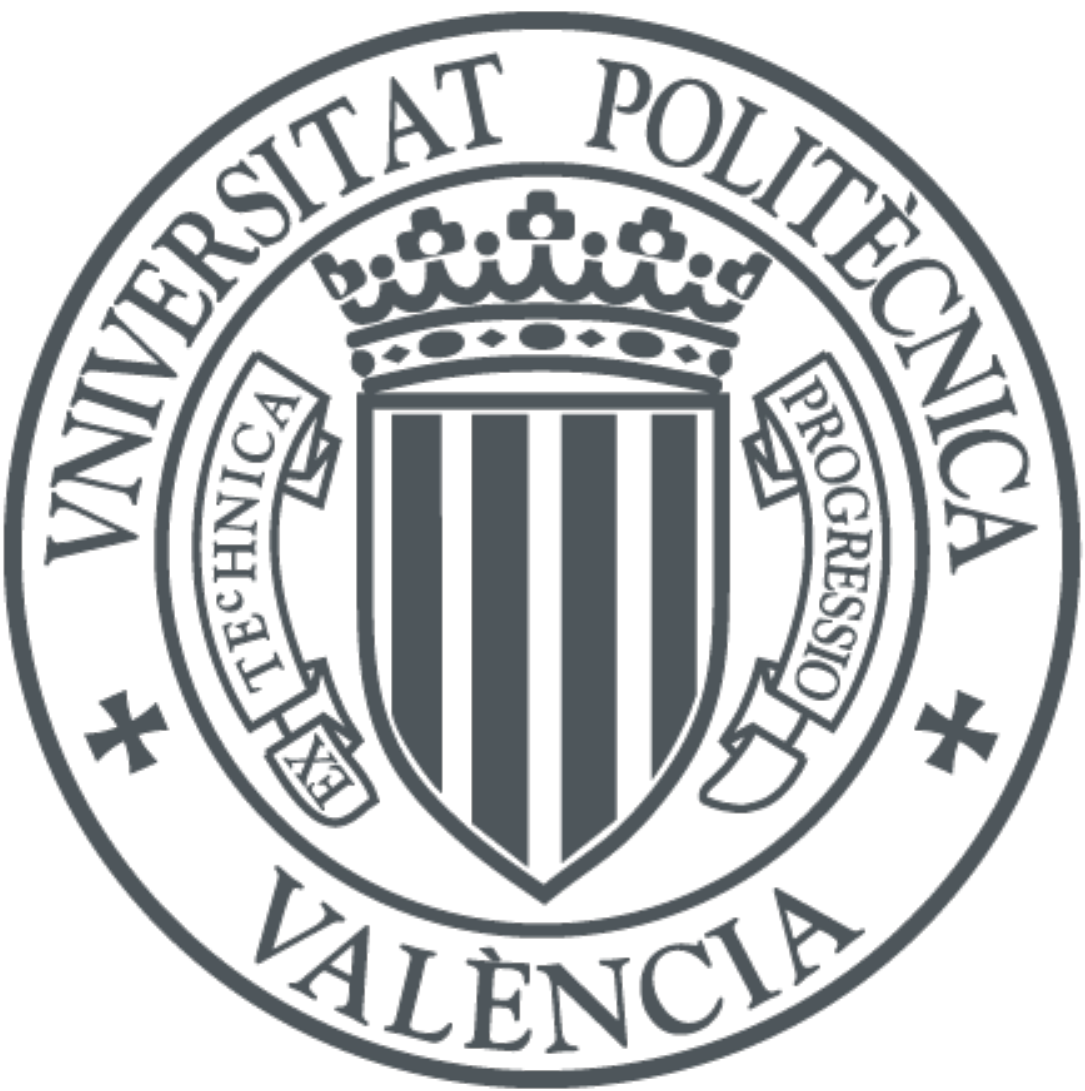

The final publication is available at

http://dx.doi.org/10.1209/0295-5075/111/34002

Copyright European Physical Society

Additional Information 


\title{
Geometrical isotropy in perforated plates with subwavelength holes decorated with Archimedean patterns
}

\author{
V. Gómez-Lozano ${ }^{1}$, C. Rubio ${ }^{1}$, P. Candelas ${ }^{1}$, F. Belmar $^{1}$ and A. Uris ${ }^{1, *}$ \\ 1 Centro de Tecnologías Físicas: Acústica, Materiales y Astrofísica, Universitat Politècnica de València, Camino de \\ Vera s/n, 46022 Valencia. SPAIN. \\ * e-mail: auris@fis.upv.es
}

PACS $43.20 .+\mathrm{g}-$ General linear acoustics

PACS 43.35. $+d-$ Ultrasonics, quantum acoustics and physical effects of sound

PACS 42.79.Dj - Gratings

\begin{abstract}
The design and use of small apertures perforated in opaque plates to control the transmission of ultrasonic waves has been widely studied in recent years. The ultrasonic transmission response of brass plates perforated with Archimedean patterns of subwavelength hole arrays immersed in water is reported, both numerically and experimentally, in this work. It is shown that an increase in the geometrical isotropy of the elementary cells of the Archimedean patterns gives rise to a suppression of both minimum and maximum transmission corresponding to the destructive and constructive interferences, leading to uniformity within the angle-dependent transmitted sound power coefficient. The experimental results are in close agreement with the calculated ones. This property can be used to design ultrasonic devices such as filters and sensors.
\end{abstract}

Introduction. - The extraordinary optical transmission through metal film perforated with a periodic array of subwavelength holes was reported by Ebbesen et al. [1] in 1998. Since then, the phenomenon of extraordinary optical transmission has been intensively studied both theoretically and experimentally due to the potential applications that could be developed [2]. The findings for electromagnetic waves were transferred to acoustic waves motivated by the nature of the waves regarding light and sound. In recent years, the study of acoustic transmission through subwavelength apertures has received considerable attention and has been investigated both theoretically and experimentally.

The extraordinary acoustic transmission was first suggested theoretically [3] and later experimentally confirmed for a grating of subwavelength slits [4] and a twodimensional hole array [5] with the Fabry-Perot resonances inside the holes being the main contribution to the transmission peaks. Theoretical results for subwavelength slits and square hole arrays in the rigid solid limit was also reported [6]. Apart from the extraordinary acoustic transmission, perforated plates with subwavelength holes arrays exhibit higher attenuation than predicted by the mass law [7], due to the Wood anomalies [8]. It was demonstrated that there was an interplay between Fabry-Perot resonances inside the holes, lattice resonances in periodic arrays and elastic Lamb modes on the ultrasonic transmission perforated plates with subwavelength holes inmersed in water [9]. The investigations on acoustic transmission through subwavelength apertures restricted to plates perforated with periodic arrays of apertures showed that the geometrical parameters of the hole array are important in the ultrasonic transmission through perforated plates [10-12]. Recently, many phenomena regarding the extraordinary acoustic transmission through subwavelength hole arrays have been demonstrated [13-17]

The acoustic transmission through a plate perforated with a periodic array (or semiregular tilings) of subwavelength apertures [18] showed strong resonant transmissions that were attributed to the coherent diffraction by the long-range order of the quasiperiodic structure. It was also studied on a plate with quasiperiodic surface corrugations [19].

In this letter, we studied the ultrasound transmission through plates perforated with Archimedean patterns of subwavelength holes. Archimedean tilings are constructed from regular convex polygons arranged identically around each vertex and the plane is tessellated by these polygons. Johannes Kepler was the first who gave a description of the of the eleven different Archimedean tilings, including 

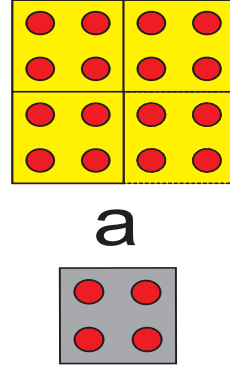
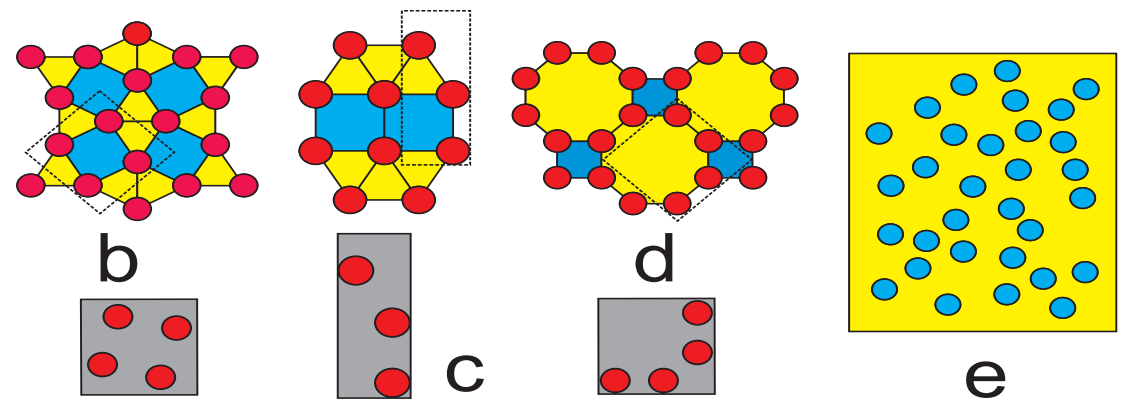

Fig.1: Structure and elementary cell for (a) periodically square distribution of circular holes, (b) Archimedean tiling (4, 3, 3, 4, 3), (c) Archimedean tiling (3, 3, 3, 4, 4), (d) Archimedean tiling (4, 8, 8) and (e) random distribution.

the traditional Bravais lattice [20]. To denote the shape and number of polygons around each identical vertex, the mathematical Grnbaum-Shephard [21] notation is used. A set of integers (n1, n2, n3, ) denotes a tiling of a vertex type in the way that n1-gon, n2-gon, n3-gon, meet consecutively on each vertex [22]. The symbol $(4,3,3,4$, 3 ) represents a tiling in which a square, two equilateral triangles, a square and a equilateral triangle gather edge to edge around a vertex. Here, we focus our analysis on how, by increasing the geometrical isotropy, that is, the disorder in the elementary cells of the Archimedean patterns, the location and amplitude of the minimum and maximum transmission vary. The variations within the angle-dependent incidence acoustic transmission and their physical origins are analysed and discussed.

Experimental set up. - The experimental set-up is based on the well known ultrasonic immersion transmission technique. This technique makes use of a couple of transmitter/receiver ultrasonic transducers. In our case, a couple of transducers with a central frequency of $250 \mathrm{kHz}$ and a frequency range between $155-350 \mathrm{kHz}$ were used. When a pulse is launched by the emitter piston transducer through the perforated plate, the signal is detected by the receiving piston transducer and acquired by the pulser/receiver, post amplified and digitalised by a digital PC oscilloscope (Picoscope model 3224). The transmission spectrum is calculated as

$$
|T(\omega)|^{2}=|H(\omega)|^{2} /\left|H_{0}(\omega)\right|^{2}
$$

from the power spectrum of the signal $H(\omega)$ normalised with the reference signal power spectrum $H_{0}(\omega)$ measured without the sample plate. The angle dependent measurements were done in angle steps of $\Delta \theta=1^{0}$ and comprising $0^{0} \leq \theta \leq 60^{\circ}$. The measurements were made using brass plates with $350 \mathrm{~mm}$ in width and $450 \mathrm{~mm}$ in length $\left(\rho_{b}=7,890 \mathrm{~kg} / \mathrm{m}^{3},\left(c_{l}\right)_{b}=5,670 \mathrm{~m} / \mathrm{s},\left(c_{t}\right)_{b}=3,230 \mathrm{~m} / \mathrm{s}\right.$ ) and $2 \mathrm{~mm}$ thickness, immersed in water $\left(\rho_{w}=1,000\right.$ $\left.\mathrm{kg} / \mathrm{m}^{3},\left(c_{l}\right)_{w}=1,480 \mathrm{~m} / \mathrm{s}\right)$.

Five different samples were used. The first one was a standard periodically square distribution of circular holes. A set of three configurations of Archimedean patterns were used. Archimedean tilings were selected in order to increase the disorder in the elementary cells. It is noted that, the disorder in the elementary cells depends on the location of the holes in those cells. The Archimedean tilings used were $(4,3,3,4,3),(3,3,3,4,4)$ and $(4,8,8)$. Finally, a sample with a random distribution of circular holes was considered. The diameter of the holes and the elementary cell dimensions were chosen to be $2.5 \mathrm{~mm}$ and $10 \times 10 \mathrm{~mm}$, respectively, except in the Archimedean tiling $(3,3,3,4,4)$ in which the hole diameter was $3 \mathrm{~mm}$ and the elementary cell dimensions were 17.18 x $6.29 \mathrm{~mm}$ with the aim to obtain structures with similar fractional apertures. In such a way, all the samples were designed to have the same fractional aperture area of about $\approx 20 \%$. Figure 1 illustrates the five different types of samples considered in this work.

Results and discussion. - The transmission sound power was calculated at the hard-solid limit, that is, assuming that the pressure field does not penetrate into the material plate because the water/plate impedance is considered infinite. The sound wave equation

$$
\left(\nabla^{2}+k^{2}\right) \phi=0
$$

where $\mathrm{k}$ is the wave number and $\phi$ is the scalar pressure, was solved splitting the space into three regions and expanding the pressure in terms of guided modes inside the hole cavities and using Rayleigh plane wave expansion on both sides of the plate. The solutions inside and outside the holes were matched at the plate surfaces, satisfying the continuity of both the field and its derivative on the hole opening and the vanishing of the normal derivative at the plate surface, resulting in a set of linear equations involving the expansion coefficients inside and outside the plate. The expansion coefficients were expressed in terms of the former and the system was reduced to a linear set of equations that were solved. Detailed expressions of the theoretical model can be found in Ref. [14]. Fig. 2 shows the angle-dependent incidence transmission properties of the Archimedean patterns by calculating the transmitted sound power coefficient, $\tau$, as a function of the normalised incident wave vector $k_{0} a / \pi$ and its component parallel to 


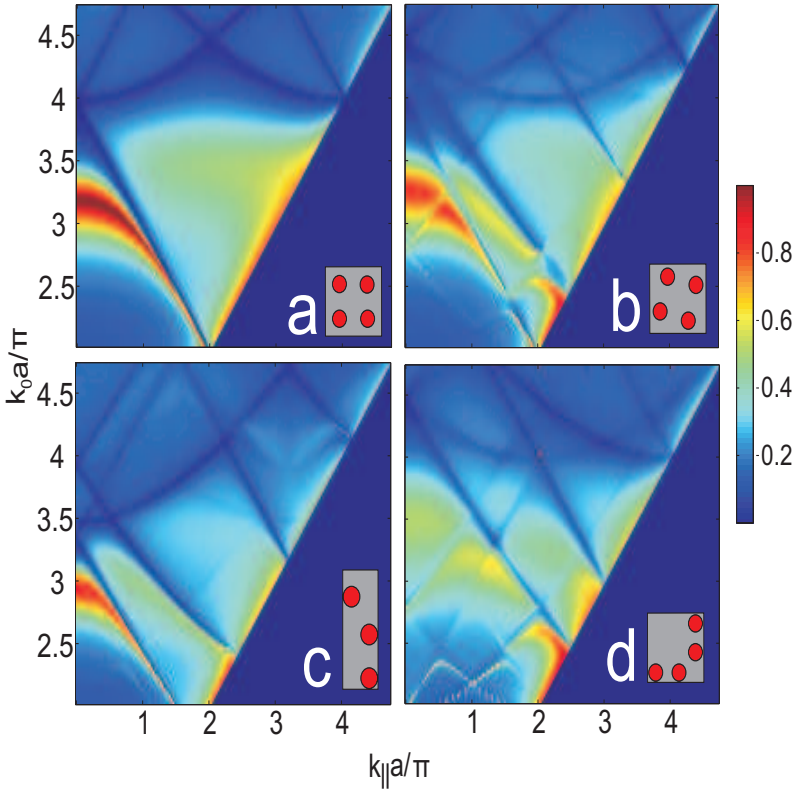

Fig.2: Calculated transmitted sound power coefficient, $\tau$, as a function of the normalised incident wave vector $k_{0} a / \pi$ and its component parallel to the plate $k_{\|} a / \pi$. (a)

Periodically square distribution of circular holes, (b)

Archimedean tiling (4, 3, 3, 4, 3), (c) Archimedean tiling $(3,3,3,4,4)$ and (d) Archimedean tiling $(4,8,8)$

the plate $k_{\|} a / \pi$, where $a$ is the lattice constant of the elementary cell.

The plate perforated with a standard periodically square distribution of circular holes (Fig. 2a) shows the Fabry-Perot full transmission at $k_{0} a / \pi=3.3$ and the Wood anomaly minimum at $k_{0} a / \pi=4$, which is evident when the incidence angle is varied. The fact that the holes are rotated causes an increase of the transmission dips, due to the fact that they depend on the location of the holes within the elementary cell, since the number of holes is the same in both cases (Fig 2a-Fig2b). In turn, these transmission dips interact with the transmission peak associated with the Fabry-Perot resonance, as shown in Fig. $2 \mathrm{~b}$. This increase in the isotropy causes a gradual increase of transmission dips and a decrease in the intensity of the Fabry-Perot resonance. This decrease is due to the incoherent interaction of resonances that occur in each hole. By varying the number of holes and reducing the symmetry of the hole distribution in the elementary cells, we obtain Archimedean tilings (3, 3, 3, 4, 4) (Fig. 1c) and (4, 8, 8) (Fig. 1d). From Fig. 2c and 2d one can find that the interplay between the minima and maxima present in the spectra is more complex than for standard periodically square distribution and it clarifies the fact that the disorder in the elementary cells results in an increase of minimum transmission and an intensity decrease of the maximum transmission related to the Fabry-Perot resonances.

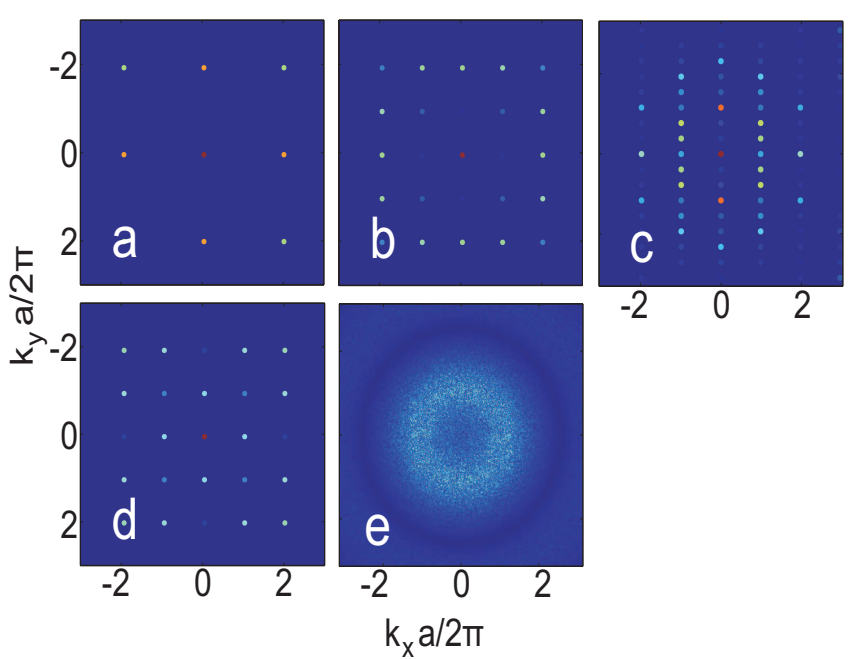

Fig.3: The geometric structure factor for (a) periodically square distribution of circular holes, (b) Archimedean tiling $(4,3,3,4,3)$, (c) Archimedean tiling (3, 3, 3, 4, 4), (d) Archimedean tiling $(4,8,8)$ and (e) random distribution.

In order to gain more insight through further investigation of this transmission behaviour, we apply the $2 \mathrm{D}$ Fast Fourier Transform to the real space lattice to obtain the structure factor of the samples considered. The results are given in Fig. 3a-d. It must be noted that in Archimedean tiling (3, 3, 3, 4, 4) (Fig. 3c), the lattice constant chosen to represent the geometric structure factor was $\sqrt{a_{x} \cdot a_{y}}=10.39 \mathrm{~mm}$. It is observed that as the geometrical isotropy of the elementary cells increases, the number of bright points increases too, which corresponds to an increase in the transmission dips or minimum transmission originated from the destructive interferences. The maximum isotropy is achieved with a random distribution of holes. This extreme situation corresponds to a maximum number of transmission dips. In such a way, a broad distribution of bright spots is reached in the reciprocal space (Fig. 3e).

The angle-dependent incidence transmitted sound power coefficient measured, $\tau$, as a function of the normalised frequency $k_{0} a / \pi$ and the parallel wave vector $k_{\|} a / \pi$, is observed from Fig. 4a-4e. The measurements are in close agreement with the calculated results even though there are some differences between them. The measured transmissions show three kinds of modes interacting with the lattice resonances: $S_{0}$ Lamb mode, $A_{0}$ Lamb mode and Scholte-Stoneley mode [24]. These modes were not predicted by the calculated results due to the fact that they were carried out with a hard-solid model in which the elastic movement of the plate was not considered. The Scholte-Stoneley mode propagates across the fluid-solid interface and it is not distinguishable in the measurements due to this mode is mixed with the A0 mode, which corresponds to the high transmission region near the angular 


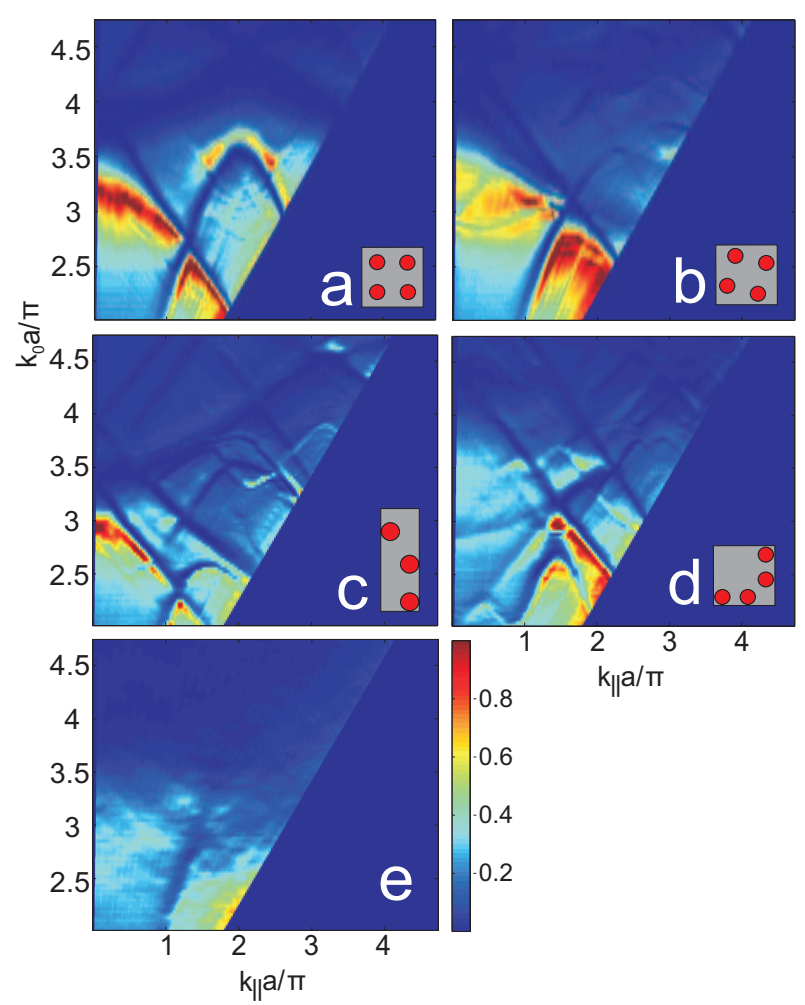

Fig.4: Transmitted sound power coefficient measured, $\tau$, as a function of the normalised incident wave vector $k_{0} a / \pi$ and its component parallel to the plate $k_{\|} a / \pi$. (a)

Periodically square distribution of circular holes, (b) Archimedean tiling (4, 3, 3, 4, 3), (c) Archimedean tiling $(3,3,3,4,4)$, (d) Archimedean tiling (4, 8, 8) and (e) random distribution..

limit of the experiment. The measured transmissions show the $S_{0}$ Lamb mode interacting with the lattice resonances that were not predicted by the calculated results due to the fact that they were carried out with a hard-solid model in which the elastic movement of the plate coupled with the surrounding fluid was not considered. The limit case corresponds to a perforated plate with random distribution of holes. In this situation a uniformity in the transmission maps is achieved, since the suppression of both the minimum and maximum transmission corresponding to the destructive and coherent interferences occurs (Fig. 4e). The results show that, as the disorder in the elementary cells increases, the cooperative effect in the transmission of the holes becomes complicated, so the constructive interference of diffracted waves from different holes in an elementary cell is reduced, leading to a lower transmission and uniformity in the transmission maps.

Conclusions. - Ultrasound transmission through perforated plates with the Archimedean pattern has been studied. The experimental results are in close agreement with the calculated ones. The results show that an increase in the geometrical isotropy of the elementary cells gives rise to an increase of minimum transmission and an intensity decrease of the Fabry-Perot maximum, leading to a homogenisation in the angle-dependent transmitted sound power coefficient. This effect is explained by a reduction in the constructive interference of diffracted waves from different holes in the elementary cells. Therefore, it is possible to achieve transmission maps without pronounced transmission peaks and dips by increasing the geometrical isotropy and this could be applied to design some new types of ultrasonic devices that may meet demands in practical applications.

$$
* * *
$$

This work has been supported by the Spanish MICINN (MAT2010-16879) and Generalitat Valenciana (PROMETEOII/2014/026).

\section{REFERENCES}

[1] Ebbesen T.W. , Lezec H. J., Ghaemi H. F., Thio T. and Wolf P.A., Nature, 391 (1998) 667.

[2] Garcia-Vidal F.J., Martin-Moreno L., Ebbesen T.W. and Kuipers L., Rev. Mod. Phys., 82 (2010) 729.

[3] Zhou L., and Kriegsmann G. A., J. Acoust. Soc. Am., 121 (2007) 3288.

[4] Hou B., Mei J., Ke M., Wen W., Liu Z., Shi J. and Sheng P., Phys. Rev. B, 76 (2007) 054303.

[5] Lu M., Liu X., Feng L., Li J., Huang C., Chen Y., Zhu Y., Zhu S. and Ming N., Phys. Rev. Lett., 99 (2007) 174301.

[6] Christensen J., Martin-Moreno L. and GarciaVidal F.J., Phys. Rev. Lett., 101 (2008) 014301.

[7] Estrada H., Candelas P., Uris A., Belmar F., García de Abajo F.J. and Meseguer F., Phys. Rev. Lett., 101 (2008) 084302.

[8] Wood R. W., Phil. Mag., 4 (1902) 396-402.

[9] Estrada H., García de Abajo F.J., Candelas P., Uris A., Belmar F. and Meseguer F., Phys. Rev. Lett., 102 (2009) 144301.

[10] Estrada H., Candelas P., Uris A., Belmar F., Meseguer F. and García de Abajo F.J., Appl. Phys. Lett., 93 (2008) 011907.

[11] Estrada H., Candelas P., Uris A., Belmar F., García de Abajo F.J. and Meseguer F., Appl. Phys. Lett., 95 (2009) 051906.

[12] Uris A., Gómez-Lozano V., Candelas P. and Belmar F., Acta Acust united Ac., 100 (2014) 595.

[13] D’Aguanno G., Le K.G., Trimm R., Alu A., Mattiucci N., Mathias A.D., Akuzbek N. and Bloemer M.J., Sci. Rep., 2 (2012) 340.

[14] Hao R., Qui CY., Ye YT., Li CH., Jia H., Ke MZ. and Liv Z., Appl. Phys. Lett., 101 (2012) 021910.

[15] Elayouch A., Addouche M., Herth E. and Khelif A., Appl. Phys. Lett., 103 (2013) 082504.

[16] Akuzbek N., Mattiucci N., Bloemer M.J., SAnghadasa M. and D'Aguanno G., Appl. Phys. Lett., 104 (2014) 161906.

[17] Wei PJ., Liu F., Liang ZX., Xu Y., Chu ST. and Li JS., Eur. Phys. Lett., 109 (2015) 14004. 
[18] Hao R., Jia H., Ye Y., Liu F., Qiu C., Ke M., and LiU Z., Eur. Phys. Lett., 92 (2010) 24006.

[19] Hao R., Qiu C., Hu Y., Tang K. and Liu Z., Phys. Lett. A, 375 (2011) 4081.

[20] Senechal M., Quasicrystals and Geometry,(Cambridge U. Press, Cambridge), 1995.

[21] Grumbaum B. and Shephard G., Tiling and Patterns,(W. H. Freeman and Company, New York),1987.

[22] Ueda K., Dotera T. and Gemma T., Phys. Rev. B., 75 (2007) 195122.

[23] Estrada H., Gomez-Lozano V., Uris A., Candelas P., Belmar F. and Meseguer F., J.Phys.: Condens. Matter, 23 (2011) 135401.

[24] Estrada H., Candelas P., Belmar F., Uris A., García de Abajo F.J. and Meseguer F., Phys. Rev. B., 85 (2012) 174301. 